\title{
Aortic remodeling after hybrid provisional extension to induce complete attachment aortic repair of chronic residual type I aortic dissection
}

\author{
Chun-Yang Huang, MD, ${ }^{\mathrm{a}, \mathrm{b}}$ Hung-Lung Hsu, MD, ${ }^{\mathrm{b}, \mathrm{c}}$ Po-Lin Chen, MD, ${ }^{\mathrm{a}, \mathrm{b}}$ Tzu-Ting Kuo, MD, ${ }^{\mathrm{a}, \mathrm{b}}$ \\ I-Ming Chen, MD, PhD, ${ }^{a, b}$ Chiao-Po Hsu, MD, PhD, ${ }^{\mathrm{a}, \mathrm{b}}$ and Chun-Che Shih, MD, $\mathrm{PhD}^{\mathrm{a}, \mathrm{b}}$
}

\section{ABSTRACT}

Objectives: Our objective was to examine the role of the provisional extension to induce complete attachment (PETTICOAT) aortic dissection repair technique with bare metal stents (BMSs) in abdominal remodeling of residual DeBakey type I aortic dissection.

Methods: We retrospectively reviewed the records of patients with chronic aneurysm formation and residual DeBakey type I aortic dissection (ie, original acute aortic dissection DeBakey type I after primary surgical open repair) who underwent arch reoperation with frozen elephant trunk replacement or endovascular debranching with or without an abdominal BMS between December 2006 and December 2016. We analyzed diameters and volumes in 3 dimensions for the true and false lumens of the thoracic and abdominal aorta as well as the thrombosis percentage of the false lumen between the non-BMS (non-PETTICOAT) and BMS (PETTICOAT) groups.

Results: Forty-seven patients who had completed at least 1 year of follow-up were included. The non-BMS (without abdominal BMS) and BMS groups had significant differences in abdominal true lumen diameter and volumetric change at the first postoperative examination and at the examination 1 year after surgery (26.8 $\mathrm{mL}$, median [19.4-34.1 mL, interquartile range (IQR)]) in non-BMS vs $42.5 \mathrm{~mL}$, median [31.1-57.9 mL, IQR]) in BMS (postoperative survey [ $F$ test, $33.775 ; P=.000])$ and $(30.1 \mathrm{~mL}$, median $[20.5-34.1 \mathrm{~mL}, \mathrm{IQR}]$ in non-BMS vs $46.6 \mathrm{~mL}$, median [31.3-57.4 mL, IQR]) in BMS (12-month survey [ $F$ test, $14.001 ; P=.001])$. The abdominal false lumen thrombosis percentage was higher in the BMS group than in the non-BMS group (25.6\%, median [16.4\%-58.9\%, IQR] in non-BMS vs $54.0 \%$, median $[36.7 \%-65.3 \%$, IQR] in the BMS group ( $F$ test, 6.318; $P=.016$ ).

Conclusions: Following reintervention for chronic residual DeBakey type I aortic dissection, PETTICOAT abdominal dissection BMS effectively expanded the thoracic and abdominal true lumen and augmented false lumen thrombosis percentage during the first postoperative year. (J Thorac Cardiovasc Surg 2019;158:1007-16)

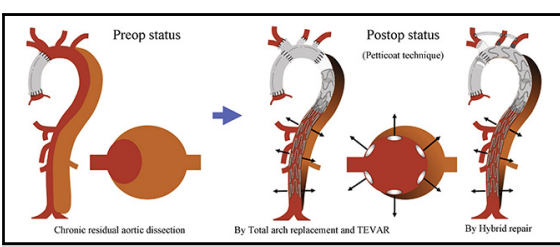

Bare metal stent supports abdominal aortic remodeling in residual type I aortic dissection.

\section{Central Message}

Thoracic stent grafting extended with a bare metal stent could be effectively used to dilate abdominal true lumen and augment false lumen thrombosis in residual DeBakey type I aortic dissection

\section{Perspective}

The proposed PETTICOAT bare metal sten (BMS) effectively dilated abdominal true lumen, augmented false lumen (FL) thrombosis, and led to further FL regression in chronic residual aortic dissection in the first year after reintervention. The findings of this study suggest that a BMS may be feasible not only in acute scenarios, but also for remodeling in cases of chronic aortic dissection.

See Commentaries on pages 1017 and 1019.
Chronic aneurysm formation is reported to develop in $15 \%$ to $50 \%$ of residual DeBakey type I aortic dissection cases. ${ }^{1}$ Reoperation is challenging and results in $19.9 \%$ overall

\footnotetext{
From the ${ }^{\mathrm{a}}$ Division of Cardiovascular Surgery, Department of Surgery, Taipei Veterans General Hospital, Taipei, Taiwan; ${ }^{\mathrm{b}}$ Institute of Clinical Medicine, School of Medicine, National Yang-Ming University, Taipei, Taiwan; and ${ }^{\mathrm{c}}$ Division of Cardiovascular Surgery, Far Eastern Memorial Hospital, Taipei, Taiwan.

Supported by grants from the Taiwan National Science Council (MOST105-2314-B010-048-MY3, MOST105-2314-B-075-006, and MOST106-2314-B-101-056) and Taipei Veterans General Hospital (VGH-104-C-117, VGH-105C-101, VGH-V106B-022，VGH-V106D24-004-MY2-1， VGH-V106D24-004-MY2-2, VGH-V107B-002, VGH-107C-147, and VGH-V105D18-001-MY3-3).
}

morbidity and $4.8 \%$ mortality. ${ }^{2}$ Numerous types of arch reoperation exist, including combinations of total arch replacement techniques or hybrid endovascular arch debranching

\footnotetext{
Received for publication May 6, 2018; revisions received Nov 27, 2018; accepted fo publication Dec 10, 2018; available ahead of print Feb 15, 2019.

Address for reprints: Chun-Che Shih, MD, PhD, Division of Cardiovascular Surgery, Taipei Veterans General Hospital, \#201 Section 2, Shih-Pai Rd, Taipei, Taiwan (E-mail: profccshih@gmail.com). $0022-5223 / \$ 36.00$

Copyright $(2018$ by The American Association for Thoracic Surgery https://doi.org/10.1016/j.jtcvs.2018.12.041
} 


\section{Abbreviations and Acronyms \\ BMS = bare metal stent \\ CT $=$ computerized tomography \\ FL $\quad=$ false lumen \\ PETTICOAT $=$ provisional extension to induce complete attachment \\ ROI $=$ region of interest \\ SINE $\quad=$ stent graft-induced new entry \\ TEVAR $=$ thoracic endovascular aortic repair \\ $\mathrm{TL}=$ true lumen}

Scanning this $\mathrm{QR}$ code will take you to the article title page to access supplementary information.

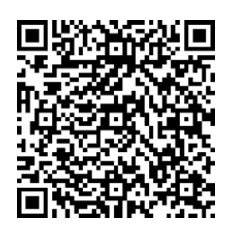

with descending thoracic endovascular aortic repair (TEVAR). ${ }^{3}$ Midterm and long-term aortic remodeling is critical after reintervention. However, prominent aortic remodeling is usually limited to the thoracic segment covered by the stent graft, and few reports have provided data on outcomes for the abdominal aortic portion. ${ }^{4-6}$ In our previous study, significant abdominal portion remodeling after TEVAR was found, resulting from radial force propagation from thoracic stent grafts. However, the duration was at least 3 years if no stent graft-induced new entry (SINE) occurred. ${ }^{4}$ Therefore, to improve the prognoses of patients with residual aortic dissection DeBakey type I more efficiently, surgical consideration should address not only thoracic dissection aneurysm extinction but also abdominal aortic remodeling. The provisional extension to induce complete attachment (PETTICOAT) procedure is an ideal technique for this purpose because it combines thoracic stent grafting and an abdominal bare metal stent (BMS). ${ }^{7}$ In addition to improving thoracic remodeling associated with the use of different types of arch reintervention with thoracic stent grafting, the PETTICOAT procedure might induce abdominal true lumen (TL) expansion and false lumen (FL) thrombosis with regression; this possibility was mentioned by Sobocinski colleagues. $^{8}$ The PETTICOAT procedure provides scaffold support directly and contributes radial force for the abdominal portion of the aorta. However, these previous findings regarding the PETTICOAT procedure were limited to acute or subacute events. The clinical outcomes of chronic conditions, especially residual aortic dissection DeBakey type I, are lacking. The objective of the present study was to determine the clinical outcomes and morphologic changes of residual aortic dissection after the PETTICOAT procedure had been conducted. Diameters and volumes were analyzed to assess TL and FL changes of the thoracic and abdominal aortae, and 3-dimensional analysis software was used to determine the proportions of FL thrombosis during 1-year observation. ${ }^{4}$ Patients who did not undergo the PETTICOAT procedure were included in the analysis for comparison.

\section{MATERIALS AND METHODS}

A retrospective review was conducted in which 347 patients who underwent endovascular treatment for aortic dissection between December 2006 and December 2016 at Taipei Veterans General Hospital were identified. Sixty-two patients with chronic aneurysm formation from residual DeBakey type I aortic dissection (after primary surgical aortic repair of acute DeBakey type I aortic dissection) who had received reoperation were enrolled in this study. All enrolled patients required arch reintervention either through total arch replacement or hybrid endovascular arch debranching combined with descending aorta stenting grafting. Patients were divided into BMS and non-BMS groups according to whether extended abdominal BMSs had been implanted. Aortic remodeling was compared between groups after at least 1 full year. The re-exploration indications were progressive aneurysmal expansion to a maximum diameter $>6 \mathrm{~cm}$ or an annual increase in diameter of more than $0.5 \mathrm{~cm}$ with maximal size $>5 \mathrm{~cm}$ during consecutive observations. The distal landing zone margin of each thoracic stent graft was above the diaphragm to avoid paraplegia. Since 2014, dissection BMSs have been commercially available in Taiwan; such BMSs normally deploy after proximal stent grafting and extend sequentially to the aortic bifurcation with at least 2 segments overlapping for each pair of adjacent components. All patients' preoperative characteristics and demographic characteristics were documented. The study was approved by the institutional review board of our institute.

\section{Surgical Procedures}

Total arch replacement (with modified frozen elephant trunk technique) and TEVAR. The right axillary artery and common femoral artery were exposed and looped. Double arterial cannulae were set individually. The innominate and left common carotid arteries were clamped after consecutive transection. An Ultramax Y graft (Atrium Medical, Hudson, $\mathrm{NH}$ ) was used for relocation of arch branches. The bilateral limbs of the $\mathrm{Y}$ graft were anastomosed to the transected innominate and left carotid artery in an end-to-end fashion.

After ascending aortic clamping, cardiac arrest was achieved under antegrade cardioplegia infusion. The ascending aorta was opened, and pathologic tissue was removed. When the rectal temperature cooled to $25^{\circ} \mathrm{C}$, systemic circulation was arrested, and the axillary arterial cannula supplied a perfusion of bilateral cerebrum hemispheres under a continuous flow of $15^{\circ} \mathrm{C}$ cold blood $(1000 \mathrm{~mL} / \mathrm{min})$. A straight Ultramax graft of a suitable size was modified into a reverse shape and applied for reconstruction of the total arch and elephant trunk. After complete reconstruction, femoral arterial inflow was restarted. The proximal reverse sleeve graft was anastomosed to the vascular graft of the former ascending replacement for primary emergent aortic repair. Subsequently, the main trunk of the Y graft was anastomosed to the reverse sleeve graft or former ascending replaced graft. A thoracic stent graft was then introduced from the femoral artery. The size of the thoracic stent graft was determined according to the elephant trunk graft diameter, with $20 \%$ to $30 \%$ oversizing.

Hybrid endovascular arch debranching and TEVAR (hybrid surgery). Arch debranching was performed according to proximal landing zone requirements (ie, carotid-subclavian artery bypass for zone 2, carotid-carotid bypass for zone 1, aortobicarotid bypass for zone 0 , or a combination of these procedures and supra-aortic branch chimney or fenestration techniques). Size selection was based on diameter 


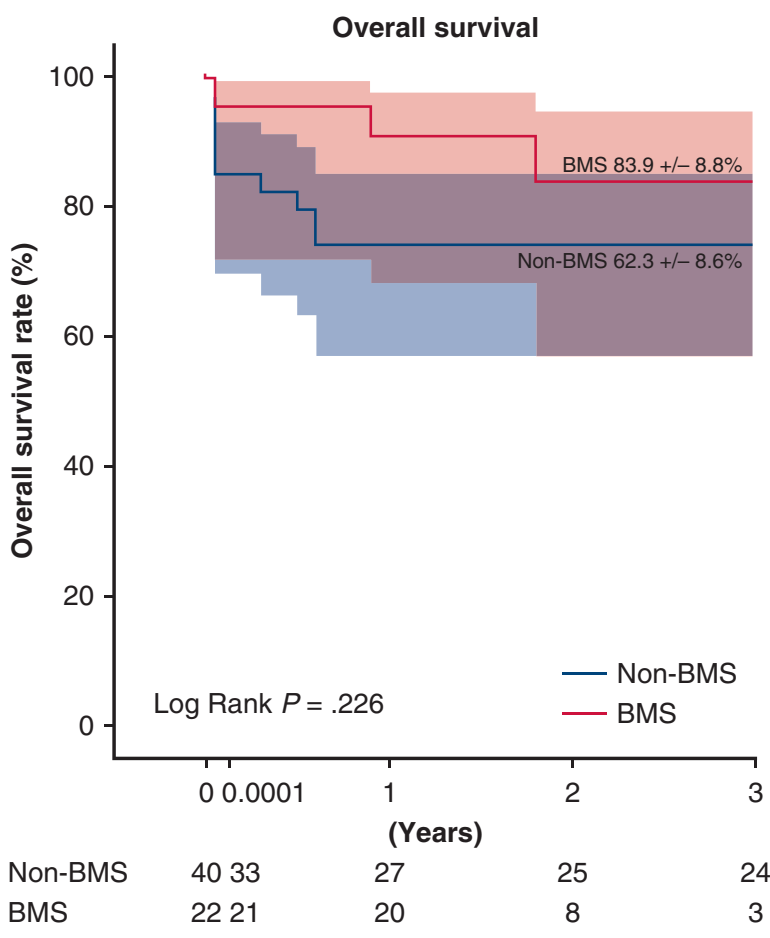

A

FIGURE 1. Comparison of clinical follow-up between non-bare metal stent group (Non-BMS) and bare metal stent group $(B M S)$ after reinterventiona procedure for chronic residual DeBakey type I aortic dissection. Time varying outcome with shaded areas showing $95 \%$ confidence intervals based on Kaplan-Meier estimation of Non-BMS group (blue) versus BMS group (red). A, Survival rate ( $x$-axis, 0.001 mean hospital mortality time). B, Freedom from stent graft-induced new entry $($ SINE) rate.

of former ascending aorta graft in primary emergent aortic repair with $20 \%$ to $30 \%$ oversizing. The thoracic stent graft was introduced through femoral access in a retrograde fashion. The bypassed left subclavian was occluded

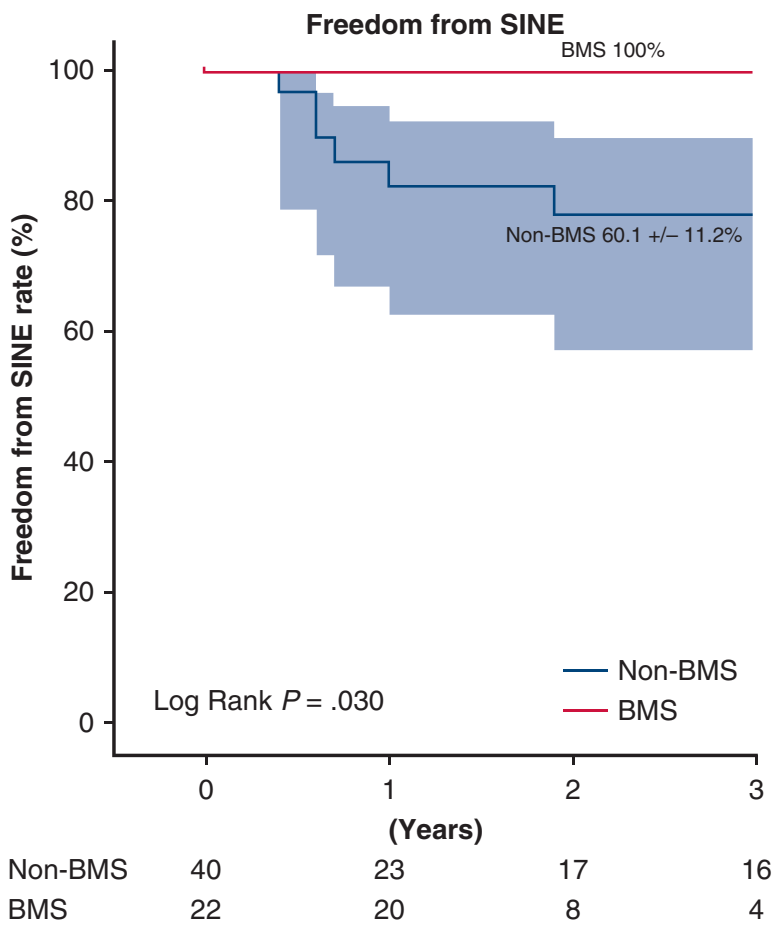

B
outcome with shaded areas showing $95 \%$ confidence intervals based on
A, Survival rate (x-axis, 0.001 mean hospital mortality time). B, Freedom with coils or through plug embolization for the zone 2 procedure, and the proximal carotid artery was subsequently occluded with surgical ligation for the zone 1 procedure.

TABLE 1. Demographic and preoperative characteristics

\begin{tabular}{|c|c|c|c|}
\hline Characteristic & No bare metal stent $(n=27)$ & Bare metal stent $(n=20)$ & $P$ value \\
\hline \multicolumn{4}{|l|}{ Demographic } \\
\hline Age $(y)$ & $62(55-67)$ & $53(45.3-67.8)$ & .143 \\
\hline Gender (M/F) & $22 / 5$ & $14 / 6$ & .489 \\
\hline DM & 2 & 2 & 1.00 \\
\hline Hyperlipidemia & 4 & 3 & 1.00 \\
\hline Hypertension & 22 & 15 & .723 \\
\hline Coronary artery disease & 5 & 4 & 1.00 \\
\hline Chronic renal failure & 1 & 3 & .298 \\
\hline Old cerebrovascular disease & 7 & 2 & .266 \\
\hline Marfan syndrome & 3 & 4 & .438 \\
\hline Smoking & 10 & 8 & .836 \\
\hline \multicolumn{4}{|l|}{ Preoperative } \\
\hline Chronicity $(\mathrm{y})^{*}$ & $10.2(6.5-12.7)$ & $8.9(3.7-10.9)$ & .156 \\
\hline Branch from false lumen & $3(1-3)$ & $3(2-3.8)$ & .292 \\
\hline Residual tear number & $3(2-4)$ & $4(3-5)$ & .062 \\
\hline \multicolumn{4}{|l|}{ Thoracic status } \\
\hline Thrombosis & 19/7/1 (70.4/25.9/3.7) & $12 / 3 / 5(60.0 / 15.0 / 25.0)$ & .078 \\
\hline Regression & 25/1/1 (92.6/3.7/3.7) & 18/1/1 (90.0/5.0/5.0) & .952 \\
\hline \multicolumn{4}{|l|}{ Abdominal status } \\
\hline Thrombosis & 20/6/1 (74.1/22.2/3.7) & $13 / 7 / 0(65.0 / 35.0 / 0)$ & .384 \\
\hline Regression & 25/1/1 (92.6/3.7/3.7) & 19/1/0 (95.0/5.0/0) & .560 \\
\hline
\end{tabular}

Values are presented as median (interquartile range) or $\mathrm{n}$ (partial $\mathrm{n} /$ total $\mathrm{n} / \%$ ). $D M$, diabetes mellitus. ${ }^{*}$ Mean follow-up time after primary exploration. 
TABLE 2. Perioperative characteristics and postoperative complication

\begin{tabular}{|c|c|c|c|}
\hline Characteristic or complication & No bare metal stent $(\mathbf{n}=\mathbf{2 7})$ & Bare metal stent $(n=20)$ & $P$ value \\
\hline \multicolumn{4}{|l|}{ Perioperative characteristic } \\
\hline Reoperation procedure & & & .007 \\
\hline Total arch replacement + TEVAR surgery & 16 & 4 & \\
\hline Hybrid surgery (chimney or fenestration) & 11 & 16 & \\
\hline Stent brand* & & & .037 \\
\hline Cook & 17 & 18 & \\
\hline Gore & 6 & 2 & \\
\hline Medtronic & 4 & 0 & \\
\hline No. of covered stents & & & 282 \\
\hline 1 & 12 & 13 & \\
\hline 2 & 11 & 4 & \\
\hline 3 & 4 & 3 & \\
\hline Stent graft oversizing $\dagger$ & $2.0(1.2-3.1)$ & $1.4(0.7-2.1)$ & .045 \\
\hline Covered stent length $(\mathrm{cm})$ & $20.8(19.3-24.3)$ & $20.4(19.2-21.8)$ & .490 \\
\hline \multicolumn{4}{|l|}{ Postoperative complication } \\
\hline Heart failure & 0 & 0 & - \\
\hline Respiratory failure & 3 & 2 & 1.00 \\
\hline Stroke & 2 & 0 & .500 \\
\hline Acute renal failure & 2 & 0 & .500 \\
\hline Limb ischemia & 1 & 1 & 1.00 \\
\hline Paraplegia & 2 & 1 & 1.00 \\
\hline Hospital days (d) & $26(13-44)$ & $21(13-38)$ & .539 \\
\hline \multicolumn{4}{|l|}{ Follow-up 1 year } \\
\hline SINE event & $4 / 27$ & $0 / 20$ & .126 \\
\hline \multicolumn{4}{|l|}{ Thoracic status } \\
\hline Thrombosis & $0 / 3 / 24(0 / 11.1 / 88.9)$ & 0/3/17 (0/15.0/85.0) & 1.00 \\
\hline Regression & $13 / 10 / 4(48.1 / 37.0 / 16.7)$ & $11 / 4 / 5(55.5 / 20.0 / 25.0)$ & .397 \\
\hline \multicolumn{4}{|l|}{ Abdominal status } \\
\hline Thrombosis & $8 / 14 / 5(29.6 / 51.9 / 18.5)$ & $2 / 13 / 5(10.0 / 65.0 / 25.0)$ & .241 \\
\hline Regression & $23 / 1 / 3(85.2 / 3.7 / 11.1)$ & $17 / 2 / 1(85.0 / 10.0 / 5.0)$ & .537 \\
\hline \multicolumn{4}{|l|}{ Follow-up period } \\
\hline Mean follow up time from primary to re-exploration $(\mathrm{y})$ & $6.8(2.2-9.2)$ & $6.2(1.3-9.2)$ & .966 \\
\hline Mean follow-up time after re-exploration (y) & $4.4(2.9-5.4)$ & $1.8(1.3-2.8)$ & .000 \\
\hline
\end{tabular}

Values are presented as median (interquartile range), $\mathrm{n} / \mathrm{N}$, or $\mathrm{n}$ (partial $\mathrm{n} /$ total $\mathrm{n} / \%$ ). TEVAR, Thoracic endovascular aortic repair; SINE, stent graft-induced new entry. *Cook Medical, Bloomington, Ind; W.L. Gore and Associates, Flagstaff, Ariz; Medtronic, Minneapolis, Minn. †Oversizing $=($ area of distal stent graft/area of distal thoracic true lumen) -1 .

\section{Abdominal BMS}

The device was deployed and overlapped in succession through the same femoral access as the thoracic stent grafting from its distal end to the aortic bifurcation (Zenith Dissection Endovascular Stent, Cook Inc, Bloomington, Ind).

Surveillance protocol. All patients were subjected to computed tomography (CT) surveillance and received their first scan within 1 month after the procedure, followed by a repeat scan after 6 months and yearly scans thereafter. The patents were required to visit our clinic regularly every 3 months after discharge. All scan images were uploaded and saved for further evaluation. Telephone interviews were used to confirm the status of patients not attending follow-up for more than 6 months.

\section{Diameter and Volumetric Investigation}

CT was performed using a Hi-Speed Advantage scanner (GE Healthcare, Waukesha, Wis) with a $5-\mathrm{mm}$ collimation setting; $10-\mathrm{mm} / \mathrm{sec}$ table speed; pitch $=2$; and $120-\mathrm{kV}, 230$ - to $250-\mathrm{mA}$ tube current. The entire aorta, from the aortic arch to the aortic bifurcation, was divided into its thoracic and abdominal portions at the celiac trunk level, as previously described. ${ }^{4-7}$
Diameter and volume on CT delayed-phase imaging were measured, including TL, FL, and total lumen. Digital imaging and communications in the medical data were transferred to OsiriX MD version 1.1 (Pixmeo, Geneva, Switzerland) for measurement. Volumes were automatically calculated using region-of-interest-computed volumes.

Diameter and volume changes in corresponding aortic levels were compared for preoperative and first postoperative surveillance and for preoperative and 12-month surveillance within groups. The intergroup diameter and volume changes were also compared.

\section{Assessment of FL Thrombosis and Regression}

In assessment of FL thrombosis status, the extent was recorded as none, partial, or complete thrombosis within the covered endograft or BMS portion. The extent of regression was evaluated with a methodology similar to that used to assess thrombosis status. ${ }^{9,10}$ After visible contrast flow was excluded, the thrombus percentage of the FL was also calculated using the following equation: ${ }^{11,12}$

Thrombus $\%=[1-($ contrast - in - FL volume $/$ FL volume $)] \times 100 \%$ 
TABLE 3. Thoracic portion alteration during first-year surveillance

\begin{tabular}{|c|c|c|c|c|c|c|c|c|c|}
\hline Variable & Preoperative survey & Postoperative survey & $P$ value $*$ & $F$ test & $P$ value $\dagger$ & 12-mo survey & $P$ value* & $F$ test & $P$ value $\dagger$ \\
\hline \multicolumn{10}{|l|}{ Diameter } \\
\hline \multicolumn{10}{|l|}{$\mathrm{TL}(\mathrm{cm})$} \\
\hline Non-BMS & $1.69(1.35-2.47)$ & $2.87(2.43-3.33)$ & .000 & 3.697 & .061 & $3.13(2.83-3.65)$ & .000 & 2.138 & .151 \\
\hline BMS & $2.55(1.47-3.29)$ & $2.81(2.44-3.23)$ & .004 & & & $3.24(2.75-3.68)$ & .000 & & \\
\hline \multicolumn{10}{|l|}{$\mathrm{FL}(\mathrm{cm})$} \\
\hline Non-BMS & $3.68(2.47-5.11)$ & $3.11(2.26-3.95)$ & .000 & 0.384 & .539 & $2.20(0.91-3.57)$ & .000 & 2.882 & .097 \\
\hline BMS & $3.07(1.24-3.92)$ & $2.74(0.76-3.42)$ & .001 & & & $2.03(0.63-3.68)$ & .004 & & \\
\hline \multicolumn{10}{|l|}{ Total $(\mathrm{cm})$} \\
\hline Non-BMS & $5.72(4.85-6.51)$ & $6.22(5.08-6.95)$ & .014 & 1.574 & .216 & $5.52(4.69-6.54)$ & .866 & 0.869 & .356 \\
\hline BMS & $5.24(3.75-6.39)$ & $5.78(3.62-6.66)$ & .232 & & & $5.65(4.02-6.63)$ & .086 & & \\
\hline \multicolumn{10}{|l|}{ Volume (mL) } \\
\hline \multicolumn{10}{|l|}{$\mathrm{TL}(\mathrm{cm})$} \\
\hline Non-BMS & $83.5(66.2-128.9)$ & $120.3(99.7-175.2)$ & .000 & 1.246 & .270 & $164.3(123.7-186.2)$ & .000 & 0.111 & .740 \\
\hline BMS & $129.0(72.7-188.0)$ & $167.9(130.4-188.2)$ & .001 & & & $174.4(149.6-201.3)$ & .000 & & \\
\hline \multicolumn{10}{|l|}{ FL (cm) } \\
\hline Non-BMS & $311.3(159.8-369.5)$ & $307.5(156.7-510.1)$ & .063 & 0.000 & .985 & $269.3(93.5-421.0)$ & .002 & 2.487 & .122 \\
\hline BMS & $323.7(85.6-456.9)$ & $328.2(66.6-442.1)$ & .093 & & & $344.7(51.2-458.7)$ & .391 & & \\
\hline \multicolumn{10}{|l|}{ Total $(\mathrm{cm})$} \\
\hline Non-BMS & $398.1(298.2-658.0)$ & 420.9 (298.7-619.4) & .200 & 0.021 & .886 & $377.1(283.1-601.7)$ & .581 & 1.747 & .193 \\
\hline BMS & $443.0(234.2-679.7)$ & $510.9(240.9-605.5)$ & .086 & & & $544.7(228.6-650.6)$ & .411 & & \\
\hline \multicolumn{10}{|l|}{ Thrombosis $(\%)$} \\
\hline Non-BMS & $46.3(28.0-67.1)$ & $88.6(78.8-94.4)$ & .000 & 5.412 & .025 & $90.6(79.1-96.5)$ & .000 & 0.953 & .334 \\
\hline BMS & $66.9(43.2-86.6)$ & $84.3(54.8-97.6)$ & .014 & & & $94.6(74.5-98.9)$ & .001 & & \\
\hline
\end{tabular}

Values are presented as median (interquartile range). $T L$, True lumen; $B M S$, bare metal stent; $F L$, false lumen; Non-BMS, no bare metal stent. *Compared with preoperative survey. $\dagger$ By analysis of variance $(d f[1,44])$.

\section{Statistical Analysis}

All variables presented in the tables are expressed as median (interquartile range [IQR]). The Mann-Whitney $U$ test was used to compare continuous variables between groups, and the Fisher exact test was used for nominal variables preoperatively. Analysis of covariance was used to compare continuous variables between groups immediately postoperatively and at 1-year follow-up. Kaplan-Meier analysis was used to calculate survival rates and freedom from SINE occurrence for each year. Data were analyzed using SPSS statistical software version 22.0 (IBM-SPSS Inc, Armonk, NY).

\section{RESULTS}

The arch reoperation procedure (total arch replacement with frozen elephant trunk technique or hybrid endovascular arch debranching through chimney or fenestration methods) (Figure 3A-D) was completed in 62 patients. Eight surgical mortalities comprised 6 with postoperative hypovolemic shock, 1 with brain stem infarction, and 1 with pneumonia complicated by septic shock. After first-year follow-up, 3 patients lost contact, and 4 died from aortoesophageal fistula with massive bleeding, stent graftrelated infection with septic shock, pneumonia with respiratory failure, and brain tumor with metastasis. Proportions of 1 -year survival in the non-BMS and BMS groups were $74.0 \%$ and $90.9 \%$, respectively (Figure $1, A$ ). The remaining 47 patients had received regular follow-up CT for more than 1 year. Among them, 27 patients did not receive abdominal
BMS (non-BMS group); the other 20 patients underwent composite abdominal BMS (BMS group). The demography exhibited no significant differences between groups (Table 1). With regard to perioperative characteristics, a lesser degree of distal stent graft oversizing occurred in the BMS group relative to the non-BMS group (median, 2.0; IQR, 1.2-3.1 in the non-BMS group vs median, 1.4; IQR, 0.7-2.1 in the BMS group; $P=.045$ ) (Table 2). Additionally, more patients in the BMS group received hybrid endovascular arch debranching procedures (11 out of 27 in the non-BMS group vs 16 out of 20 in BMS group; $P=.007$ ) and Cook TX2 TAA endovascular graft implantations (17 out of 27 in the non-BMS group vs 18 out of 20 in BMS group; $P=.037)$ relative to those in the non-BMS group (Table 2). No significant differences in postoperative complications were found between groups (Table 2). Four patients in the non-BMS group and no patients in the BMS group experienced SINE during the first year after these procedures. After the first year, the SINE occurrence rate exhibited a significant difference between the 2 groups (log-rank $P=.03$ ) (Figure 1, $B$ ). The median postoperative follow-up periods were 4.4 years for the non-BMS group and 1.8 years for the BMS group, representing a significant difference (4.4 years [IQR, 2.9-5.4 years] in the non-BMS group vs 1.8 years [IQR, 1.3-2.8 years] in the BMS group; $P=.000)$ (Table 2). 


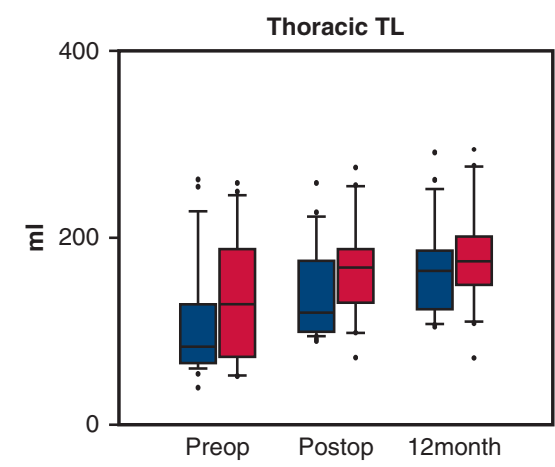

A

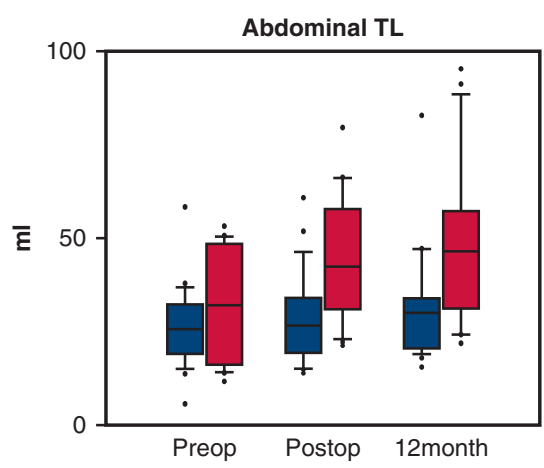

D

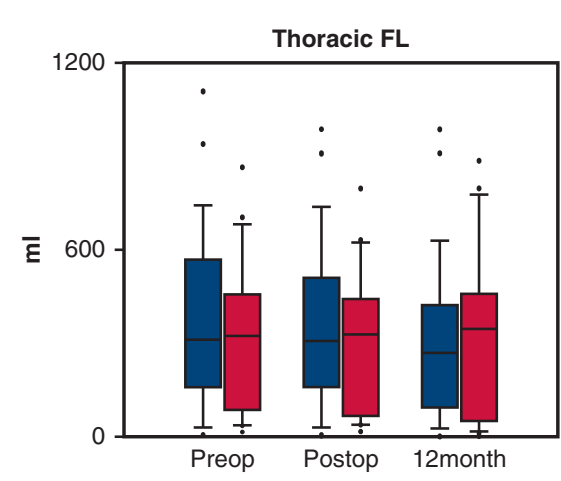

B

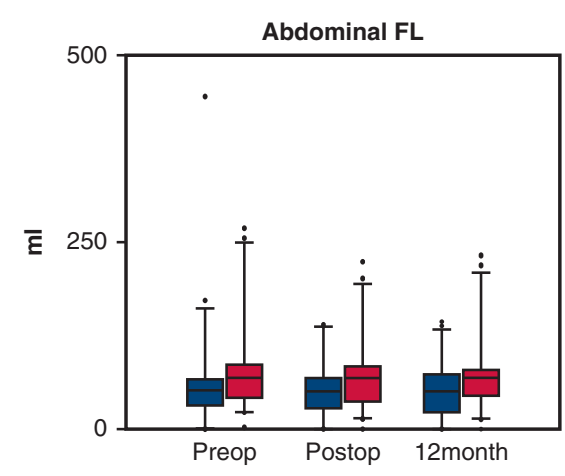

E

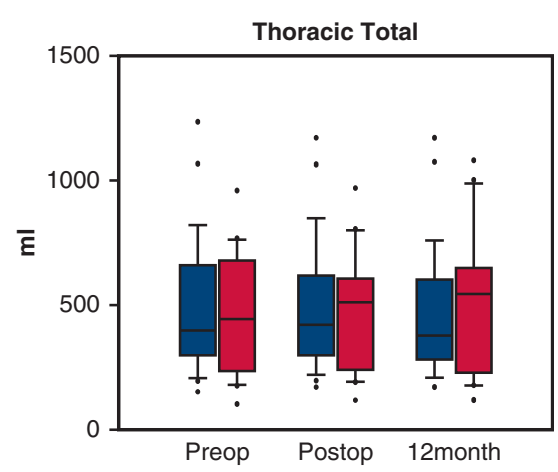

C

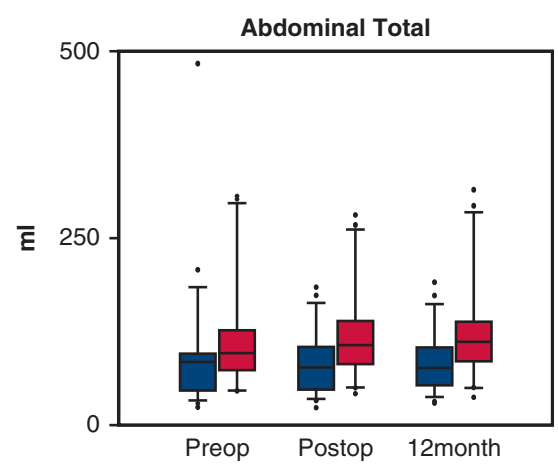

F

FIGURE 2. Thoracic and abdominal aortic volume remodeling at preoperative, postoperative, and 1-year observation between non-bare metal stent group (blue) and bare metal stent group (red). A, Thoracic true lumen volume (in milliliters). B, Thoracic false lumen volume (in milliliters). C, Thoracic total volume (in milliliters). D, Abdominal true lumen volume (in milliliters). E, Abdominal false lumen volume (in milliliters). F, Abdominal total volume (in milliliters). The upper and lower borders of the box indicate the upper and lower quartiles. The middle horizontal line indicates the median. The upper and lower whiskers indicate the maximum and minimum values of nonoutliers. Extra dots indicate outliers. Preop, Preoperative; Postop, postoperative; $T L$, true lumen; $F L$, false lumen.

\section{Aortic Remodeling}

Thoracic portion. Analyses of patients within groups revealed significant thoracic TL expansion and FL remodeling, but between groups, patients displayed similar changes of diameters and volumes relative to those from preoperative calculations, and the expansions remained stable through 12 months after repair (Table 3 and Figure 2, $A$ and $B$ ). The non-BMS group exhibited greater immediate increases in FL thrombosis formation than did the BMS group from preoperative to postoperative survey (88.6\% [IQR, $78.8 \%-94.4 \%$ ] in the non-BMS group vs $84.3 \%$ [IQR, $54.8 \%-97.6 \%$ ] in the BMS group; $F$ test $=5.412 ; P=.025$ ) (Table 3). However, the FL thrombosis percentage continued to increase in the BMS group; it finally reached a percentage similar to that of the non-BMS group in the 12-month survey (Table 3).

Abdominal portion. Significant postoperative expansion of the abdominal TL was observed in the BMS group but not in the non-BMS group; in non-BMS-group patients, the abdominal TL remained similar from preoperation to postoperation, as indicated in analyses of both diameter $(1.24 \mathrm{~cm}$ [IQR, $0.97-1.24 \mathrm{~cm}]$ in the non-BMS group vs
$1.75 \mathrm{~cm}$ [IQR, $1.40-2.21 \mathrm{~cm}$ ] in the BMS group; $F$ test $=28.809 ; P=.000)$ and volume $(26.8 \mathrm{~mL}[\mathrm{IQR}$, 19.4-34.1 mL] in the non-BMS group vs $42.5 \mathrm{~mL}$ [IQR, 31.1-57.9 $\mathrm{mL}]$ in the BMS group; $F$ test $=33.775$; $P=.000$ ) (Table 4 and Figure $2, D$ ). In addition, at 12 months the lumen expanded significantly more in the BMS group than in the non-BMS group in diameter $(1.21 \mathrm{~cm}$ [IQR, $1.12-1.60 \mathrm{~cm}$ ] in the non-BMS group vs $1.69 \mathrm{~cm}$ [IQR, $1.40-2.33 \mathrm{~cm}$ ] in the BMS group; $F$ test $=37.348 ; P=.000)$ and volume $(30.1 \mathrm{~mL}$ [IQR, 20.5-34.1 mL] in the non-BMS group vs $46.6 \mathrm{~mL}$ [IQR, 31.3-57.4 mL] in the BMS group; $F$ test $=14.011$; $P=.001$ ] (Table 4, Figure 2, $D$, and Figure 3, $A-F$ ). Neither group manifested significant FL reduction from preoperative to postoperative or after 12-month survey measurements, according to analyses of both diameter and volume (Table 4, Figure 2, $E$, and Figure 3, $A-F$ ). A higher proportion of thrombosis was observed in the BMS group than in the non-BMS group in the 12-month survey $(25.5 \%$ [IQR, $16.4 \%-58.9 \%$ ] in the non-BMS group vs $54.0 \%$ $[\mathrm{IQR}, 36.7 \%-65.3 \%$ ] in the BMS group; $F$ test $=6.318$; $P=.016$ ) (Table 4 and Figure $3, A-F)$. 
TABLE 4. Abdominal portion alteration during first-year surveillance

\begin{tabular}{|c|c|c|c|c|c|c|c|c|c|}
\hline Variable & Preoperative survey & Postoperative survey & $P$ value* & $F$ test & $P$ value $\dagger$ & 12-mo survey & $P$ value* & $F$ test & $\boldsymbol{P}$ value $\dagger$ \\
\hline \multicolumn{10}{|l|}{ Diameter } \\
\hline \multicolumn{10}{|l|}{$\mathrm{TL}(\mathrm{cm})$} \\
\hline Non-BMS & $1.23(0.94-1.60)$ & $1.24(0.97-1.24)$ & .275 & 28.809 & .000 & $1.21(1.12-1.60)$ & .146 & 37.348 & .000 \\
\hline BMS & $1.39(0.91-1.78)$ & $1.75(1.40-2.21)$ & .000 & & & $1.69(1.40-2.33)$ & .000 & & \\
\hline \multicolumn{10}{|l|}{$\mathrm{FL}(\mathrm{cm})$} \\
\hline Non-BMS & $1.47(0.34-1.97)$ & $1.52(0.40-2.01)$ & .166 & 2.865 & .098 & $1.56(0.34-1.86)$ & .848 & 1.539 & .221 \\
\hline BMS & $1.71(0.85-2.20)$ & $1.51(0.94-2.25)$ & .171 & & & $1.40(0.90-2.22)$ & .167 & & \\
\hline \multicolumn{10}{|l|}{ Total $(\mathrm{cm})$} \\
\hline Non-BMS & $2.55(2.13-2.90)$ & $2.65(2.27-2.94)$ & .166 & 7.521 & .009 & $2.62(2.17-2.99)$ & .848 & 4.187 & .047 \\
\hline BMS & $3.36(2.38-3.55)$ & $3.59(2.64-3.95)$ & .171 & & & $3.64(2.69-3.84)$ & .167 & & \\
\hline \multicolumn{10}{|l|}{ Volume } \\
\hline \multicolumn{10}{|l|}{$\mathrm{TL}(\mathrm{mL})$} \\
\hline Non-BMS & $25.8(19.1-32.5)$ & $26.8(19.4-34.1)$ & .061 & 33.775 & .000 & $30.1(20.5-34.1)$ & .002 & 14.011 & .001 \\
\hline BMS & $32.2(16.2-48.5)$ & $42.5(31.1-57.9)$ & .000 & & & $46.6(31.3-57.4)$ & .000 & & \\
\hline \multicolumn{10}{|l|}{ FL (mL) } \\
\hline Non-BMS & $52.3(32.0-66.8)$ & $51.0(28.7-68.1)$ & .008 & 2.322 & .135 & $51.0(22.5-73.4)$ & .414 & 2.348 & .133 \\
\hline BMS & $68.5(43.1-86.6)$ & $68.4(37.3-84.1)$ & .052 & & & $68.4(44.5-79.6)$ & .232 & & \\
\hline \multicolumn{10}{|l|}{ Total (mL) } \\
\hline Non-BMS & $83.6(45.9-95.1)$ & $76.8(46.8-104.6)$ & .517 & 8.213 & .006 & $76.2(52.6-103.5)$ & .683 & 7.258 & .010 \\
\hline BMS & $95.9(72.7-126.5)$ & $106.4(80.9-139.0)$ & .057 & & & $111.1(84.6-137.8)$ & .044 & & \\
\hline \multicolumn{10}{|l|}{ Thrombosis $(\%)$} \\
\hline Non-BMS & $39.0(26.5-75.0)$ & $25.2(17.1-72.2)$ & .376 & 1.440 & .237 & $25.6(16.4-58.9)$ & .209 & 6.318 & .016 \\
\hline BMS & $39.4(25.2-72.0)$ & $39.3(28.1-64.9)$ & .334 & & & $54.0(36.7-65.3)$ & .044 & & \\
\hline
\end{tabular}

Values are presented as median (interquartile range). $T L$, True lumen; Non-BMS, no bare metal stent; $B M S$, bare metal stent; $F L$, false lumen. *Compared with preoperative survey. $\dagger$ By analysis of variance $(d f[1,44])$.

\section{DISCUSSION}

In this study, the PETTICOAT technique was performed on BMS group patients. The procedure involved thoracic reintervention and abdominal BMS implantation designed for chronic residual DeBakey type I aortic dissection. The non-BMS - or non-PETTICOAT - group received thoracic reintervention without a BMS. The results regarding proportions of patients without SINE events revealed significant differences between groups, except in the first postoperative year. SINE seldom occurred in the BMS group. This result implied that use of the PETTICOAT technique may result in lower incidence of SINE events. Dissection with a BMS can be used to align the distal edges of thoracic stent grafts and adjacent native intima of thoracic aortae. Proper alignment might substantially distribute and alleviate the influence of radial force, thereby preventing the stent graft edges from injuring the aortic intima. This might partially explain why the patients undergoing the PETTICOAT technique presented with fewer SINE events in the present study. However, multiple factors contribute to SINE. A high degree of stent graft oversizing in the non-BMS group could have elevated the risk of SINE, as mentioned in our previous study. ${ }^{13}$ Therefore, minimizing the preoperative distal area oversizing ratio using the distal small graft first procedure would also result in lower incidences of late distal
SINE. ${ }^{14} \mathrm{He}$ and colleagues ${ }^{15}$ claimed that their proposed modified PETTICOAT technique (BMS implantation before thoracic stent grafting) could prevent SINE events and requirements for secondary intervention more effectively than use of a conventional PETTICOAT procedure. However, in cases with prior deployment of a BMS, gap formation between the distal part of the thoracic stent graft and native aortic intima involves a higher risk for type $\mathrm{Ib}$ or gutter endoleak. In this study, the normal implantation sequence for dissection with a BMS exhibited comparable results to the aforementioned modified PETTICOAT technique for SINE prevention; the normal implantation sequence could be recommended for optimal PETTICOAT technique performance.

Prominent aortic remodeling is usually limited to the thoracic segment covered by the stent graft, and few reports have provided data on outcomes for the abdominal aorta. Some researchers have observed a significant increase in patients' average TL size along with a significant decrease in their FL size in both the thoracic and abdominal aorta in 2year follow-up after the PETTICOAT procedure. ${ }^{7,16-18}$ Although the cited results are promising, most relevant studies have been confined to using the procedure for complicated type B aortic dissection and patients experiencing acute events. In the homogenous population of patients with chronic residual DeBakey type I aortic 


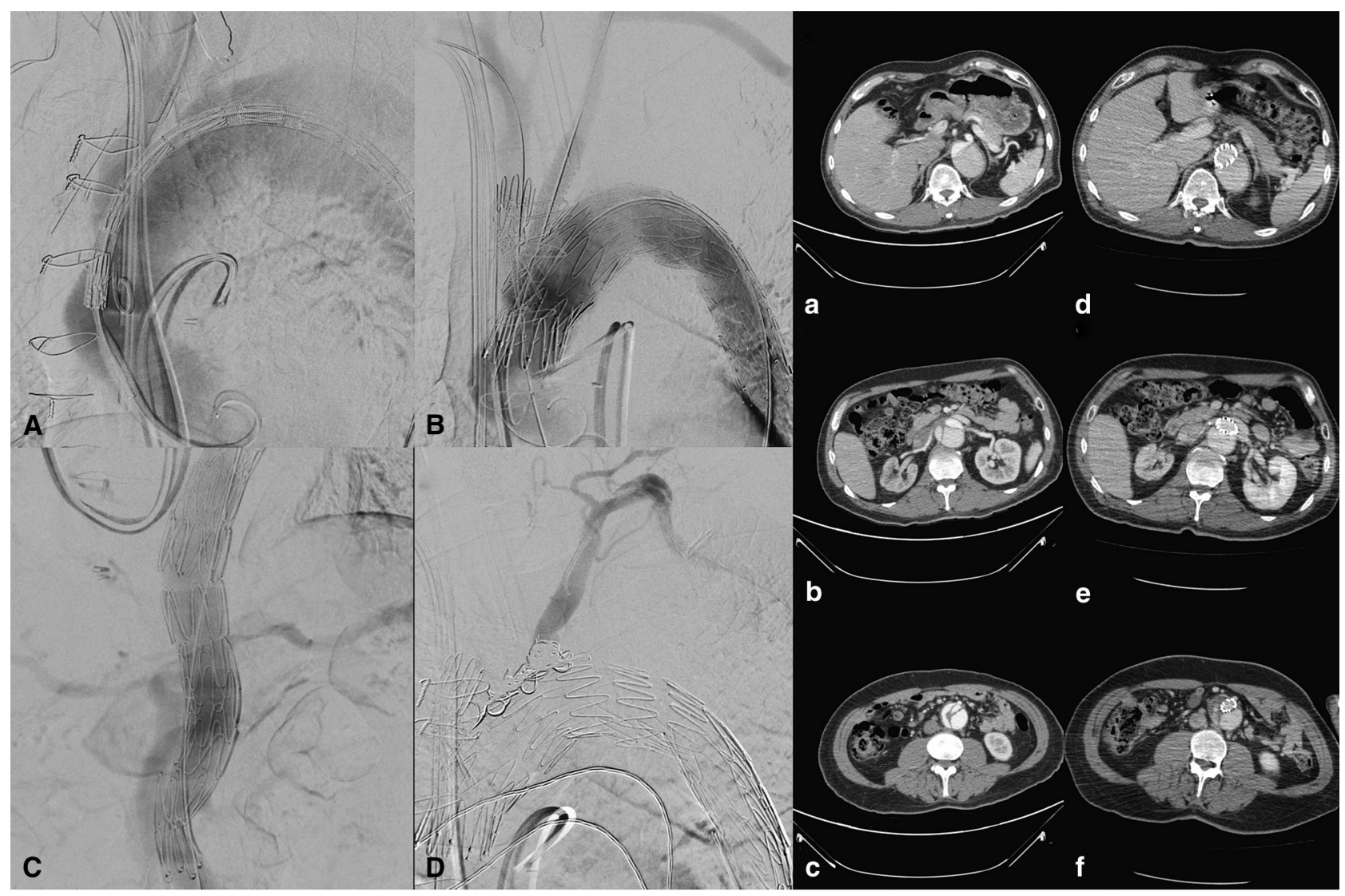

FIGURE 3. Reinterventional procedure of chronic residual DeBakey type I aortic dissection by endovascular arch chimney technique with petticoat procedure (bare metal stent group). Left carotid-subclavian artery bypass was performed before arch chimney technique. A, Supra-aortic branch stent and thoracic cover stent delivered individually. B, Innominate/left carotid artery stent and arch/descending aorta stent graft deployed simultaneously (chimney technique). C, Abdominal bare metal stent deployed sequentially (provisional extension to induce complete attachment procedure). D, Left subclavian artery was embolized by coils. Abdominal true lumen size comparison of computed tomographic imaging during preoperative and postoperative observation. a, Preoperative image of celiac trunk. b, Preoperative image of renal artery. c, Preoperative image of inferior mesenteric artery level. $\mathrm{d}$, Postoperative image of celiac trunk. e, Postoperative image of renal artery. f, Postoperative image of inferior mesenteric artery level.

dissection in this study, patients with the PETTICOAT technique exhibited thoracic remodeling similar to that of patients in the non-PETTICOAT group. Additionally, both groups displayed significant thrombosis formation of the thoracic FL in immediate postoperative examinations. These phenomena were also discussed in our previous study, in which noticeable thoracic lumen remodeling and FL thrombosis occurred after TEVAR procedure where only thoracic stent grafting was used. ${ }^{2,4}$ However, in this study, only the PETTICOAT procedure revealed significantly superior expansion of the abdominal TL in the BMS group relative to the non-BMS group. The direct radial force of a BMS might have played a crucial role in the favorable results for abdominal TL remodeling rather than indirect and slow propagation by radial force of thoracic covered stents in our previous non-BMS study group. ${ }^{4}$ On the other hand, it seems that efficient postoperative abdominal FL thrombosis was not observed in either group immediately. Notably, patients who received the PETTICOAT technique exhibited subtle but persistent increases in abdominal thrombosis percentages for a full year. Our findings differed slightly from those of other previous studies. ${ }^{7,8,19}$ Their findings claimed the PETTICOAT procedure immediately contributed to FL thrombosis with perfect regression in both thoracic and abdominal segments. However, these outcomes were confined to cases of acute and subacute aortic dissection. This implies that our study might potentially extend the PETTICOAT procedure application in abdominal FL thrombosis of chronic cases. Despite this encouraging result within 1-year observation, postoperative partial thrombosis status increased compared with preoperative status after the PETTICOAT procedure. The inadequate thrombosis status had been reported to be associated with a higher annual aortic growth rate, and poor long-term survival. ${ }^{10,20-23}$ However, the increased total thrombosis of the FL that was also observed within 1 year concomitantly and that would be beneficial for long-term survival. It implied thrombosis formation within the first year is dynamic. One year of 


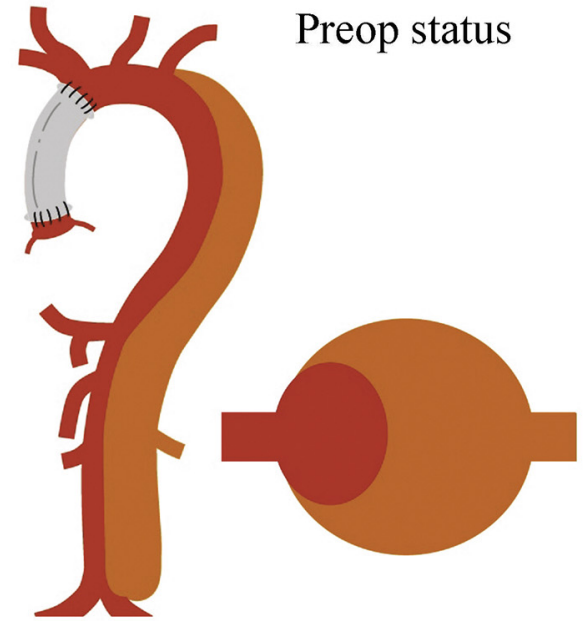

Chronic residual aortic dissection

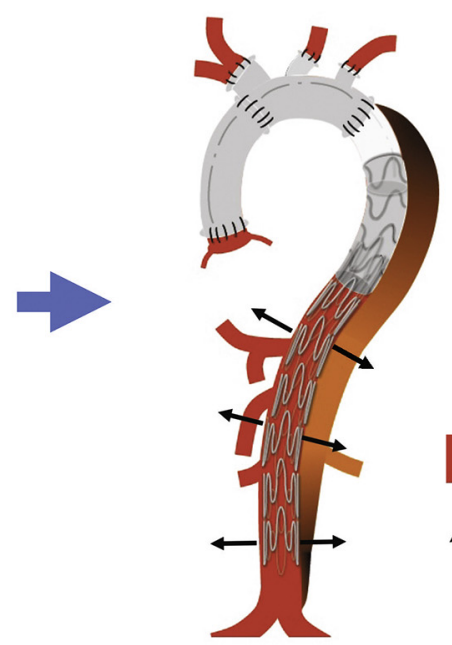

By Total arch replacement and TEVAR

\section{Postop status}

(Petticoat technique)
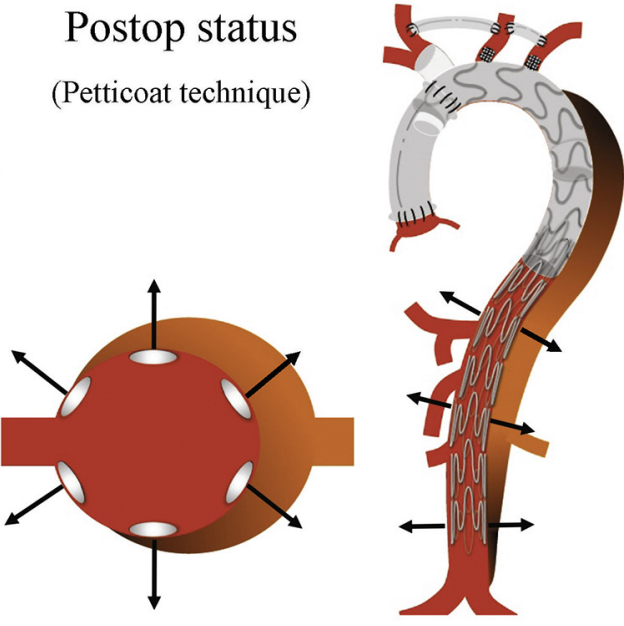

By Hybrid repair

FIGURE 4. In 1-year results, abdominal true lumen was expanded and thrombus was augmented in false lumen after reinterventional procedure for chronic residual DeBakey type I aortic dissection. There are 2 types of arch reinterventional procedures concomitant with abdominal aorta bare metal stenting involved in our study. Left, In preoperative status, small and compressed abdominal true lumen with patent false lumen of chronic residual DeBakey type I aortic dissection. Middle, In postoperative status, the provisional extension to induce complete attachment (Petticoat) effect expanded abdominal true lumen and augmented false lumen remodeling in total arch replacement with modified frozen elephant trunk technique and thoracic endovascular aortic repair (TEVAR). Right, In postoperative status, the Petticoat effect expanded abdominal true lumen and augmented false lumen remodeling in hybrid endovascular arch debranching surgery. Preop, Preoperative. Postop, postoperative.

observation was insufficient to decide the final thrombosis result. Under subtle but persistent increase in thrombosis percentage, partial thrombosis might be improved to total thrombosis over some observation period longer than 1 year. This possibility should be further explored in future studies. The PETTICOAT technique not only exhibited the benefits of SINE prevention, excellent thoracic remodeling, and superior abdominal TL expansion, but also may have forced potential FL thrombosis. These advantages of the PETTICOAT technique for chronic residual aortic dissection are also summarized in Figure 4 by 2 types of thoracic re-interventional procedures (Video 1, supplementary data).

Although total abdominal aortic diameters and volumes did not obviously shrink among the patients in either group, the expanded total aortic volume associated with the PETTICOAT technique resulted from proportional expansion of the TL as opposed to FL dilation. The status of abdominal FL remodeling remained inconclusive after the first year of postoperative observation, but the higher thrombosis percentage caused by the PETTICOAT technique was a potential beneficial factor for shrinkage. Annual observation of aortic renovation was essential. Regarding endovascular surgical technique, when cannulation of malperfused visceral branches is mandatory, the PETTICOAT procedure can create adequate TL space for angiocatheter engagement because of the wide mesh structure of a BMS. However, relatively long procedural and fluorescence times are indispensable for the PETTICOAT procedure.
This study was limited by its retrospective single-center design, small sample size of enrolled patients, and short follow-up interval, especially with the BMS group patients

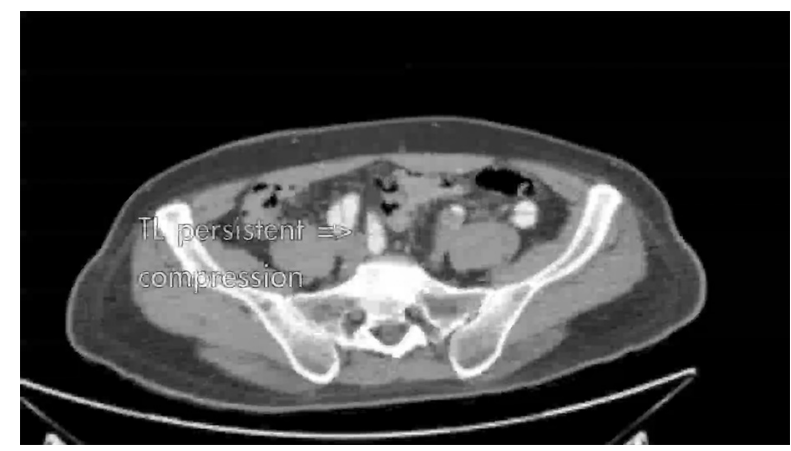

VIDEO 1. Male 49-year-old patient with chronic residual DeBakey type I aortic dissection was followed for 3 years. Thoracic aneurysm formation was prominent with maximal diameter larger than $6 \mathrm{~cm}$ in the past 6 months. Preoperative small and compressed true lumen (TL) with patent and expanded false lumen (FL) were revealed on computed tomography with 3-dimensional reconstructed image. This patient underwent hybrid endovascular arch debranching surgery with concomitant provisional extension to induce complete attachment (PETTICOAT) procedure. The thoracic-covered stent was delivered to the previous vascular graft and deployed. Afterward, an abdominal bare metal stent was deployed and overlapped from the distal end of the thoracic stent graft to the aortic bifurcation. Postoperative and 1-year follow-up imaging after the PETTICOAT procedure revealed superior TL dilation and FL remodeling in thoracic and abdominal portions relative to the non-PETTICOAT procedure. Video available at: https://www.jtcvs.org/article/S0022-5223(18)33480-9/fulltext. 
who were only followed up for a mean 2.1 years after surgery. Although remodeling in the thorax is a critical prognostic factor, the influence of the PETTICOAT BMS procedure appeared to be relatively correlated with abdominal TL remodeling and FL thrombosis. The procedure may lead to relatively low SINE incidence or extended intervals between SINE occurrences. The effects of abdominal remodeling are not fully understood, and a longer cohort study is necessary to confirm the findings of the present study.

\section{CONCLUSIONS}

For re-exploration of chronic residual aortic dissection of DeBakey type I, the PETTICOAT technique might efficiently expand abdominal TL and augment FL thrombosis during the first postoperative year. However, continuous observation is still recommended to verify the final outcomes of abdominal FL remodeling.

\section{Conflict of Interest Statement}

Authors have nothing to disclose with regard to commercial support.

The authors thank Mr. Chun-Han Shih and Mr. Chun-Kai Shih for providing technical assistance.

\section{References}

1. Zierer A, Voeller RK, Hill KE, Kouchoukos NT, Damiano RJ Jr, Moon MR. Aortic enlargement and late reoperation after repair of acute type A aortic dissection. Ann Thorac Surg. 2007:84:479-87.

2. Huang CY, Chen CW, Chen PL, Chen WY, Chen IM, Hsu CP, et al. Association between aortic remodeling and stent graft-induced new entry in extensive residual type A dissecting aortic aneurysm after hybrid arch repair. Ann Vasc Surg. 2016;31:60-9.

3. Szeto WY, Bavaria JE. Hybrid repair of aortic arch aneurysms: combined open arch reconstruction and endovascular repair. Semin Thorac Cardiovasc Surg. 2009;21:347-54.

4. Huang CY, Hsu HL, Chen PL, Chen IM, Hsu CP, Shih CC. The impact of distal stent-graft induced new entry on aortic remodeling of chronic type B dissection. Ann Thorac Surg. 2018;105:785-93.

5. Qin YL, Deng G, Li TX, Jin RW, Teng GJ. Risk factors of incomplete thrombosis in the false lumen after endovascular treatment of extensive acute type B aortic dissection. J Vasc Surg. 2012;56:1232-8.

6. Dinsmore RE, Willerson JT, Buckley MJ. Dissecting aneurysm of the aorta: aortographic features affecting prognosis. Radiology. 1972;105:567-72.

7. Lombardi JV, Cambria RP, Nienaber CA, Chiesa R, Teebken O, Lee A, et al. Prospective multicenter clinical trial (STABLE) on the endovascular treatment of complicated type B aortic dissection using a composite device design. J Vasc Surg. 2012;55:629-40.
8. Sobocinski J, Lombardi JV, Dias NV, Berger L, Zhou Q, Jia F, et al. Volume analysis of true and false lumens in acute complicated type B aortic dissections after thoracic endovascular aortic repair with stent grafts alone or with a composite device design. J Vasc Surg. 2016;63:1216-24.

9. Czermak BV, Mallouhi A, Perkmann R, Steingruber IE, Waldenberger P, Neuhauser B, et al. Serial CT volume and thrombus length measurements after endovascular repair of Stanford type B aortic dissection. J Endovasc Ther. 2004;11:1-12.

10. Tsai TT, Evangelista A, Nienaber CA, Myrmel T, Meinhardt G, Cooper JV, et al. Partial thrombosis of the false lumen in patients with acute type B aortic dissection. N Engl J Med. 2007;357:349-59.

11. Quint LE, Platt JF, Sonnad SS, Deeb GM, Williams DM. Aortic intimal tear: detection with spiral computed tomography. J Endovasc Ther. 2003;10:505-10.

12. Khoynezhad A, Walot I, Kruse MJ, Rapae T, Donayre CE, White RA. Distribution of intimomedial tears in patients with type B aortic dissection. J Vasc Surg. 2010;52:562-8.

13. Huang CY, Weng SH, Weng CF, Chen WY, Chen IM, Hsu CP, et al. Factors predictive of distal stent graft-induced new entry after hybrid arch elephant trunk repair with stainless steel-based device in aortic dissection. J Thorac Cardiovasc Surg. 2013;146:623-30.

14. Chen IM, Huang CY, Weng SH, Lin PY, Chen PL, Chen WY, et al. Implantation sequence modification averts distal stent-graft-induced new entry after endovascular repair of Stanford type B aortic dissection. J Vasc Surg. 2016;64:281-8.

15. He H, Yao K, Nie WP, Wang Z, Liang Q, Shu C, et al. Modified PETTICOAT technique with pre-placement of a distal bare stent improves early aortic remodeling after complicated acute Stanford type B aortic dissection. Eur J Vasc Endovasc Surg. 2015;50:450-9.

16. Hofferberth SC, Foley PT, Newcomb AE, Yap KK, Yii MY, Nixon IK, et al. Combined proximal endografting with distal bare-metal stenting for management of aortic dissection. Ann Thorac Surg. 2012;93:95-102.

17. Lombardi JV, Cambria RP, Nienaber CA, Chiesa R, Mossop R, Haulon S, et al. Aortic remodeling after endovascular treatment of complicated type B aortic dissection with the use of a composite device design. J Vasc Surg. 2014;59:1544-54.

18. Hofferberth SC, Newcomb AE, Yii MY, Yap KK, Boston RC, Nixon IK, et al. Combined proximal stent grafting plus distal bare metal stenting for management of aortic dissection: superior to standard endovascular repair? J Thorac Cardiovasc Surg. 2012;144:956-62.

19. Melissano G, Bertoglio L, Rinaldi E, Civlini E, Tshomba Y, Kahlberg A, et al. Volume change in aortic true and false lumen after the "PETTICOAT" procedure for type B aortic dissection. J Vasc Surg. 2012;55:641-51.

20. Trimarchi S, Tolenaar JL, Jonker FH, Murray B, Tsai TT, Eagle KA, et al. Importance of false lumen thrombosis in type B aortic dissection prognosis. J Thorac Cardiovasc Surg. 2013;145(3 Suppl):S208-12.

21. Liu F, Ge YY, Guo W, Liu XP, Jia X, Xiong J, et al. Preoperative thoracic false lumen branches are predictors of aortic enlargement after stent grafting for DeBakey IIIb aortic dissection. J Thorac Cardiovasc Surg. 2018;155: 21-9.

22. Li D, Ye L, He Y, Cao X, Liu J, Zhong W, et al. False lumen status in patients with acute aortic dissection: a systematic review and meta-analysis. J Am Heart Assoc. 2016;5:e003172.

23. Song SW, Chang BC, Cho BK, Yi G, Youn YN, Lee S, et al. Effects of partial thrombosis on distal aorta after repair of acute DeBakey type I aortic dissection. J Thorac Cardiovasc Surg. 2010;139:841-7.

Key Words: aortic dissection, aortic remodeling, bare metal stent, PETTICOAT, residual aortic dissection 